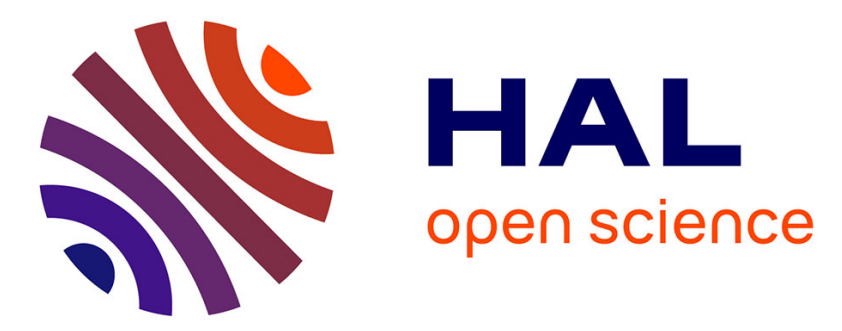

\title{
Génération et placement de couleurs sur une vue de type métro
}

Christophe Hurter, Mathieu Serrurier, Gilles Tabart, Jean-Luc Vinot

\section{To cite this version:}

Christophe Hurter, Mathieu Serrurier, Gilles Tabart, Jean-Luc Vinot. Génération et placement de couleurs sur une vue de type métro. IHM 2009, 21ème Conférence Francophone sur l'Interaction Homme-Machine, Oct 2009, Grenoble, France. pp 55-58, 10.1145/1629826.1629835 . hal-01022164

\section{HAL Id: hal-01022164 \\ https://hal-enac.archives-ouvertes.fr/hal-01022164}

Submitted on 23 Jul 2014

HAL is a multi-disciplinary open access archive for the deposit and dissemination of scientific research documents, whether they are published or not. The documents may come from teaching and research institutions in France or abroad, or from public or private research centers.
L'archive ouverte pluridisciplinaire HAL, est destinée au dépôt et à la diffusion de documents scientifiques de niveau recherche, publiés ou non, émanant des établissements d'enseignement et de recherche français ou étrangers, des laboratoires publics ou privés. 


\section{Génération et placement de couleurs sur une vue de type métro}

\author{
Christophe Hurter ${ }^{1,3,4}$ Mathieu Serrurier ${ }^{2} \quad$ Tabart Gilles ${ }^{1,2,3}$ Jean-Luc Vinot ${ }^{1,3,4}$ \\ ${ }^{1}$ ENAC \\ ${ }^{2}$ RPDMP-IRIT \\ 3 IHCS-IRIT \\ 7, av. Edouard Belin \\ 31400, Toulouse, France \\ 118 Route de Narbonne, \\ 31400 Toulouse, France \\ christophe.hurter@aviation-civile.gouv.fr \\ tabart@cena.fr \\ serrurie@irit.fr \\ ${ }^{4}$ DSNA/DTI/R\&D \\ 7, av. Edouard Belin \\ 31400, Toulouse, France \\ vinot@cena.fr
}

problem, thus we use a meta-heuristic approach to solve it. To validate our method, we apply it to the field of air traffic control with the maps of Flight Routes.

Les vues schématiques de type vue métro ont été utilisées afin de maximiser la perception des informations les plus pertinentes (lignes, arrêts dans le cas du métro) pour la visualisation de réseaux. Les approches de génération automatique de vue métro se focalisent principalement sur la structure physique du réseau, mais peu sur le choix des couleurs qui est pourtant un vecteur de discrimination visuelle. Dans cet article, nous proposons de nous intéresser aux choix et au placement des couleurs à affecter aux lignes de ce réseau. La première étape consiste à trouver autant de couleurs que de lignes à afficher. Ces couleurs doivent être d'une part perceptuellement les plus distantes possibles, et d'autre part désignables en utilisant le vocabulaire des couleurs. La deuxième étape consiste à placer ces couleurs de telle sorte que les routes les plus proches ont des couleurs les plus éloignées perpétuellement. Le positionnement des couleurs étant un problème NP-complet, nous utilisons une méthode méta heuristique pour le résoudre. Pour valider notre méthode, nous l'appliquons au domaine du contrôle aérien avec les cartes de routes aériennes.

MOTS CLES : visualisation, vue métro, contrôle aérien.

\section{ABSTRACT}

The schematic views for metro maps are used to maximize the transmision of relevant information (lines, metro stops) of network visualization. Automatic generation of metro maps focus primarily on the physical structure of the network, but little on the choice of colors which is an accurate visual discrimination. In this article, we propose to invesigate the generation and placement of colors to be assigned to lines of a network. The first step is to find as many colors as lines of the network. These colors must be perceptually as distant as possible, and available in the vocabulary of colors. The second step is to place these colors so that the closest lines have the more distant color. The positioning of colors is a NP-complete

Permission to make digital or hard copies of all or part of this work for personal or classroom use is granted without fee provided that copies are not made or distributed for profit or commercial advantage and that copies bear this notice and the full citation on the first page. To copy otherwise, or republish, to post on servers or to redistribute to lists, requires prior specific permission and/or a fee.

IHM 2009, 13-16 Octobre 2009, Grenoble, France

Copyright 2009 ACM 978-1-60558-461-4/09/10 ...\$5.00.
CATEGORIES AND SUBJECT DESCRIPTORS: H5.m. Information interfaces and presentation (e.g., HCI): Miscellaneous.

KEYWORDS: visualization, metro map, Air Traffic Controller.

\section{INTRODUCTION}

Les vues métro sont des représentations schématiques des réseaux qui représentent les différentes stations et les lignes de métro urbain [5]. La génération automatique des cartes de métro est une problématique qui reste d'actualité [8][1][9]. Le but de la génération automatique de vue est de maximiser la perception des informations les plus pertinentes (lignes, arrêts dans le cas du métro) pour la visualisation de réseaux. La plupart des méthodes de construction automatique consistent à optimiser la forme du réseau en utilisant des critères d'évaluation mathématiques que l'on essaye de minimiser (séparation des lignes, limitation des croisements, directions fixes, taux de lignes droites...). Ce type de choix de design correspond à l'utilisation des variables visuelles de positionnement dans la sémiologie graphique de Bertin [3] (positions en $\mathrm{X}$ et $\mathrm{Y}$ sur le plan d'affichage).

Cependant, d'autres variables visuelles de la sémiologie de Bertin n'ont pas été considérées dans le cadre de la production automatique de vues métro. Ces variables sont, entre autres, la « valeur» (semblable à la luminosité) et la «couleur» (semblable à la chrominance) qui correspondent à une propriété globale de couleur. Il est évident qu'associer une couleur distincte à chaque ligne augmente leur discrimination (ce qui est le cas des vues métro des transports urbains). Cependant, choisir et placer les couleurs au hasard n'est pas optimal pour la discrimination des routes pour au moins deux raisons. Toutes les couleurs n'ont pas le même potentiel de discrimination entre elles. Certaines lignes sont déjà discriminées par leur position géographique.

Dans cet article, nous proposons de nous intéresser aux choix et au placement des couleurs à affecter aux lignes d'un réseau. Nous proposons un algorithme déterministe pour générer autant de couleurs que de lignes dans le réseau. L'objectif est de produire un ensemble de couleurs 
le plus discriminant possible. Afin de faciliter la communication entre utilisateurs des vues métro, on s'impose de choisir des couleurs qui ont une correspondance dans le langage courant. On cherche ensuite à associer chacune de ces couleurs à une ligne en prenant garde de choisir des couleurs discriminantes pour les lignes proches. Afin de réaliser ce placement, on définit une mesure pour évaluer la qualité de la production. Dans ce contexte, le positionnement des couleurs étant un problème NP-complet, nous utilisons la méta heuristique du recuit simulé [6] pour le résoudre. Pour valider notre méthode, nous l'appliquons au domaine du contrôle aérien avec les cartes de routes aériennes dont la structure physique à déjà été optimisée.

\section{GENERATION D'UNE TABLE DES COULEURS}

En signalétique et dans les vues métro usuelles, le dessin et le choix des couleurs est réalisé par un designer graphique. Celui-ci crée manuellement un nuancier, spécifique au besoin précis, à partir de techniques acquises de composition et d'harmonie des couleurs. Sans vouloir atteindre un résultat comparable, nous recherchons une méthode générique satisfaisante de production de jeux de couleurs discriminantes (ensembles de n couleurs).

Générer des couleurs dans l'espace RGB, en séparant les valeurs des composants n'est pas une méthode optimale pour notre objectif: la valeur RGB ne nous renseigne pas sur la sensation de couleur perçue et la «distance » RGB de ces couleurs n'est pas homogène avec la distance perceptive. Pour définir les couleurs et mesurer efficacement leurs distances perceptives, nous utiliserons les espaces colorimétriques de la CIE (Commission Internationale de l'Eclairage) [4]. La CIE a construit de manière expérimentale des modèles chromatiques théoriques, définissant l'ensemble des couleurs perceptibles, indépendamment des matériels d'affichage, et prenant en compte la perception visuelle humaine. Le modèle CIE L*a*b* (CIELAB) est ainsi homogène avec la perception visuelle. La distance géométrique entre 2 couleurs dans l'espace $\mathrm{L}^{*} \mathrm{a}{ }^{*} \mathrm{~b} *$ correspond à leur distance perceptive et peut être calculée (CIE delta E 2000). Enfin, une variante LCH du modèle permet d'exprimer la couleur selon 3 dimensions plus intuitives : Luminosité, Chroma (saturation), et Hue (teinte). Les dimensions $\mathrm{L}, \mathrm{C}$ et $\mathrm{H}$ sont orthogonales et perceptuellement uniformes. Cependant, le modèle CIELAB a ses limites, le gamut RGB (couleurs réellement affichables) du périphérique ne permettra pas d'atteindre toutes les couleurs LCHab possibles et le modèle ne précise pas la notion de « couleur nommée ».

En utilisant le modèle CIE LCHab, nous pouvons aisément calculer un nombre quelconque de couleurs, régulièrement espacé sur l'axe $\langle\mathrm{H} »$ des teintes. Cet axe des teintes est en effet primordial pour une différenciation de sensation de couleur. Nous pouvons aussi calculer leurs contrastes relatifs $\left(\right.$ Delta $\left._{\mathrm{e}}\right)$. Mais cette approche n'est pas suffisante, car des valeurs constantes en luminosité (L) et en saturation (C) ne suffisent pas pour produire des couleurs expressives (nommables) ni garantir leur affichage dans le gamut RGB. Comme l'ont formulé Goethe et plus précisément Munsell [7], à chaque angle de teinte correspond un espace de distribution non homogène des couleurs sur les axes $\mathrm{L}$ et $\mathrm{C}$. La luminosité du jaune $(\mathrm{L}>$ $90 \%)$ et du violet $(\mathrm{L}<40 \%)$ par exemple sont très différentes. Des travaux et expérimentations sur les « couleurs nommées » [2][10], définissent leurs coordonnées «idéales ». En utilisant ces résultats et la table de Munsell [7], nous avons construit une courbe de correspondance des valeurs L de ces couleurs nommées selon l'angle $\mathrm{H}$ des teintes.

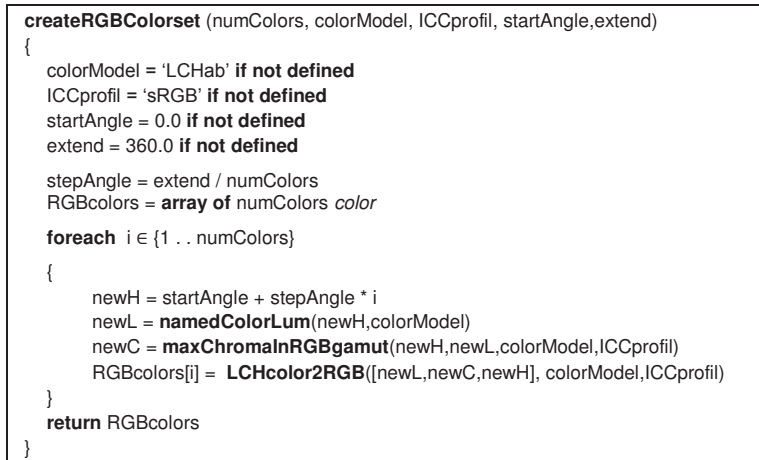

Le pseudo code ci-dessus présente la fonction de création d'un jeu de couleur RGB. Cette fonction prend en arguments le nombre de couleurs requises, le modèle de couleur (par défaut LCHab), le profil ICC cible (par défaut sRGB), ainsi qu'un angle de teinte optionnel, de « calage ». La fonction namedColorLum retourne la valeur $\mathrm{L}$ de luminosité « idéale » pour un angle $\mathrm{H}$ de teinte dans l'espace couleur spécifié. La fonction maxChromaInRGBgamut calcule la valeur $\mathrm{C}$ de saturation maximale d'une couleur d'angle $\mathrm{H}$ et de luminosité $\mathrm{L}$, dans le gamut RGB d'un périphérique cible (ICCprofil). Enfin la fonction de conversion LCHcolor2RGB retourne les valeurs RGB de la couleur LCH avec le profil ICC cible.

\section{LE PLACEMENT DES COULEURS}

En considérant que l'on possède une vue métro avec $\mathrm{N}$ lignes, la méthode décrite dans la section précédente nous permet de générer $\mathrm{N}$ couleurs distinctes. Cependant, il existe $\mathrm{N}$ ! choix d'associations possible entre $\mathrm{N}$ couleurs et $\mathrm{N}$ lignes. Il convient alors de définir une mesure de la qualité d'un placement de couleur, puis de proposer une méthode capable, si possible, de trouver le placement optimal par rapport à cette mesure. Intuitivement, on cherche à associer aux lignes les plus proches les couleurs les plus distinctes du point de vue de la perception. Nous avons besoin de définir une distance entre les couleurs. Nous avons choisi d'utiliser le Delta $\mathrm{e}_{\mathrm{e}}$ défini dans la section précédente.

Pour calculer la distance entre les lignes, on définit une ligne comme une succession de points régulièrement espacés (pour ce faire, on discrétise chaque ligne).

$$
\text { i.e. } 1_{1}=\left\{\mathrm{Pt}_{11}, \ldots \mathrm{Pt}_{1 \mathrm{~m}}\right\} \text {. }
$$

La distance classique entre deux lignes est la distance minimale entre les points des deux lignes. Cette distance 
n'est pas convenable du point de vue perceptif, car par exemple deux lignes qui se croisent perpendiculairement ont une distance nulle alors que perceptuellement elles sont très distinctes. Nous allons utiliser une distance point par point qui sera plus représentative du critère de discrimination humaine.

Soit $\mathrm{l}_{1}=\left\{\mathrm{Pt}_{11}, \ldots \mathrm{Pt}_{1 \mathrm{~m}}\right\}$ et $\mathrm{l}_{2}=\left\{\mathrm{Pt}_{21}, \ldots \mathrm{Pt}_{2 \mathrm{q}}\right\}$ avec $\mathrm{m}<\mathrm{q}$

$$
\operatorname{dist}\left(l_{1}, l_{2}\right)=\frac{\left(\sum_{i=1}^{m} \min \left(d\left(P t_{1 i}, P t_{2 j}\right), j=1 \rightarrow q\right)\right.}{m}
$$

Où d est la distance euclidienne entre deux points. Ainsi, pour chaque point de la ligne $1_{1}$, la distance de ce point à la ligne $l_{2}$ est égale à la distance minimale qui le sépare d'un point de cette ligne; la distance entre les deux lignes est alors égale à la somme de ces distances. On normalise ensuite cette distance en la divisant par la plus grande distance entre les $\mathrm{N}$ lignes.

Un placement de couleurs $\mathrm{P}$ est un ensemble de $\mathrm{N}$ couples de ligne et de couleur $\left(\mathrm{l}_{\mathrm{i}}, \mathrm{C}_{\mathrm{i}}\right)$. Nous définissons la qualité d'un placement de couleur comme suit :

Cô̂t $=\sum_{i, j, i<j}^{N} \operatorname{Delta}_{e}\left(C_{i}, C_{j}\right) \times 2 \times\left(1-\frac{1}{1+e^{-\operatorname{dist}\left(l_{i}, l_{j}\right)}}\right)$

Ce coût correspond à une moyenne pondérée des Delta $\mathrm{e}_{\mathrm{e}}$ Le poids associé à chaque Delta $_{\mathrm{e}}$ dépend de la distance entre les lignes. On applique une fonction de type sigmoïde à cette distance de sorte que son poids tende vers 1 quand la distance tend vers 0 (deux lignes proches), et vers 0 quand la distance tend vers 1 (deux lignes les plus éloignées). Ainsi l'importance du Delta $\mathrm{e}_{\mathrm{e}}$ est d'autant plus importante que les lignes sont proches.

Trouver le placement optimal parmi N ! placements possible est un problème NP complet, donc insoluble avec des méthodes déterministes. Nous utilisons la méthode du recuit simulé [6], qui est très efficace pour ce type de problème. Cette méthode consiste à partir d'une configuration initiale de la faire évoluer par voisinage. Le passage d'un état courant à son voisin se fait de deux manières : soit le voisin augmente la fonction d'évaluation auquel cas il est automatiquement accepté, soit le voisin décroît la fonction d'évaluation auquel cas il est accepté avec une probabilité qui dépend de l'amplitude de cette décroissance et d'un paramètre empirique dit de température $\mathrm{T}$ qui diminue à chaque itération. Cette méthode ne permet pas forcément de trouver la solution optimale, mais permet d'éviter les minimums locaux et ainsi de converger vers une solution acceptable.

Dans notre cas, l'espace étudié est celui du placement des couleurs préalablement calculées : un état de cet espace est un ensemble de couples [route, couleur]. On obtient un voisin en permutant les couleurs associées à deux routes. Afin de rendre le paramétrage du recuit simulé indépendant des lignes à optimiser (pour augmenter la généricité de la méthode), nous normalisons la fonction de coût.

\section{APPLICATION}

Les contrôleurs aériens utilisent dans leur environnement des systèmes de visualisation complexes qui doivent être compréhensibles avec un minimum de charge cognitive. Les avions suivent des routes aériennes (Flight Route : FR) qui sont des séquences de balises (point géographique avec une altitude). Les secteurs regroupent un ensemble de balises et de routes. Il est alors possible de faire une analogie entre les vues des routes aériennes et les plans de métro en associant une FR à une ligne et une balise à un arrêt (exemple du secteur W Figure 1).
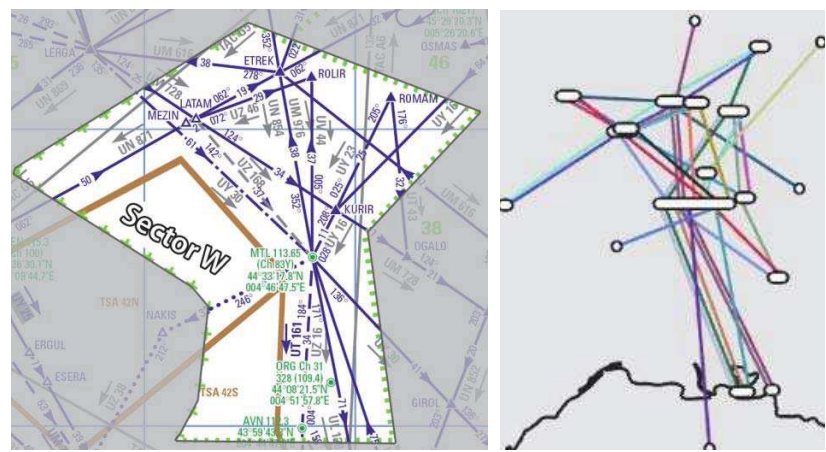

Figure 1 : Routes aériennes et leur représentation schématique associée sans optimisation

Les FR sont nombreuses et s'entremêlent, c'est pourquoi leur représentation même schématique est impossible, elles demandent trop de temps d'analyse pour des tâches du contrôle aérien par exemple la désignation et le suivi d'une route (Figure 1). Il est alors nécessaire, comme pour les plans métro, de générer une vue plus lisible. Les secteurs aériens sont nombreux et évoluent régulièrement, c'est pourquoi il n'est pas envisageable de réaliser des vues schématiques à la main. En utilisant les algorithmes d'optimisation de la structure des vues métro sur les FR, ont obtient une structure plus lisible avec un meilleur placement géographique des balises (Figure 3 de gauche).

Sur la Figure 3 de gauche, nous avons généré et placé les couleurs au hasard. Cette approche n'aide pas, voire détériore la perception des routes (confusion possible entre deux routes proches géographiquement avec des couleurs ressemblantes).

La Figure 2 utilise la même disposition des balises avec le jeu de couleurs optimisé par l'algorithme de choix de couleurs optimisé pour le nombre de routes (24 routes). Le placement est aléatoire sur la première image. On constate que par rapport au choix de couleurs au hasard on génère des couleurs plus étalées dans le spectre avec une luminosité adaptée à chaque classe de couleurs. Ces couleurs sont ainsi plus facilement nommables et différentiables.

Dans la seconde image, nous avons utilisé l'algorithme de placement des couleurs avec une fonction de coût opposée. La configuration ainsi obtenue est la moins bonne du point de vue perceptuel, par contre elle permet de va- 
lider l'efficacité de l'algorithme, car il a associé les couleurs les plus proches aux routes les plus rapprochées. Enfin, dans la Figure 3, nous utilisons un placement optimisé dans le choix des couleurs.
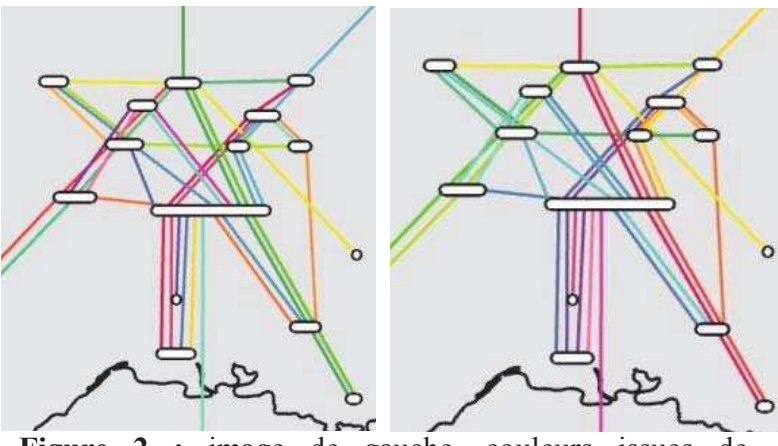

Figure 2 : image de gauche, couleurs issues de l'algorithme de génération des couleurs avec un placement aléatoire. Image de droite, placement des couleurs en associant les routes les plus proches aux couleurs les plus proches.

On constate facilement que lorsque les routes sont proches, notamment dans la partie gauche de l'image avec beaucoup de routes parallèles, les FR sont facilement différentiables. Les couleurs variant soit au niveau de la teinte, soit au niveau de la luminosité.
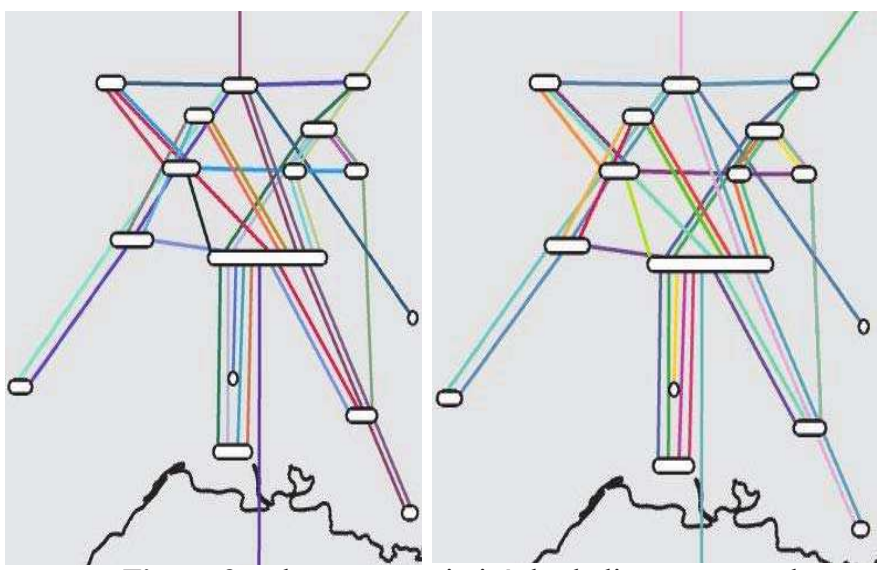

Figure 3 : placement optimisé des balises, avec couleurs choisies au hasard (image de gauche). Placement des couleurs optimales en associant les couleurs les plus éloignées perceptuellement aux routes les plus proches géographiquement (image de droite).

\section{CONCLUSIONS ET PERSPECTIVES}

Dans cet article, nous avons proposé une méthode pour générer et placer des couleurs sur des visualisations de type vue métro. Le choix et le placement de ces couleurs permettent d'améliorer la lisibilité et la compréhension de ce type de vue.

Le choix et le placement des couleurs pour les vues métro n'avaient pas été précisément étudiés jusque-là. Nous proposons une méthode originale pour générer les cou- leurs qui prennent en compte sa sémantique et sa distance perceptuelle par rapport aux autres. De plus, nous avons défini une fonction de coût qui permet de mesurer à quel point un placement de couleur est efficace. Bien que nous l'ayons appliqué au cas spécifique de la représentation des routes aériennes, il pourrait s'appliquer aussi par exemple aux cartes des transports urbains. Une perspective à court terme est de mieux contrôler la discrimination de nombreuses couleurs. En introduisant une enveloppe de variations sur les axes $\mathrm{L}$ et $\mathrm{C}$ nous pourrions augmenter le contraste de couleurs proches sur l'axe des teintes. Il serait important également d'intégrer la couleur de fond et sa luminosité dans notre algorithme de génération (dans notre exemple, certaines couleurs sont trop proches de la couleur de fond). De plus, la perception d'une couleur est variable par rapport au fond et nécessiterait des corrections type CIECAM2 [4].

Il conviendrait aussi d'appliquer notre approche à une carte de transport urbain afin et de la comparer avec celle produite par un designer. Enfin, nous envisageons de réaliser des évaluations par des utilisateurs, et ainsi mesurer l'apport respectif et combiner de l'optimisation de la structure des FR et du choix de leur couleur.

\section{BIBLIOGRAPHIE}

[1] Bekos M., Kaufmann M., Potika K., Symvonis A. : Line Crossing Minimization on Metro Maps. Proc. of Graph Drawing 2007.

[2] B. Berlin, and P. Kay, Basic color terms : their universality and evolution. Berkeley; Oxford: University of California Press (1969) pg. 196.

[3] Bertin J. : Semiology of Graphics. Editions Mouton, Gauthier-Villars, Paris, 1967.

[4] A Colour Appearance Model for Colour Management Systems: CIE CAM 2002, CIE 159, (2004)

[5] Garland K.: Mr Beck's Underground Map, Capital Transportation Publishing, 1994.

[6] Kirkpatrick S., Gelatt C. D., Vecchi M. P. : Optimization by simulated annealing. Science 220, 671 $680,1983$.

[7] Munsell, A. H. (1912). "A Pigment color System and Notation". The American Journal of Psychology.

[8] Nöllenburg, N. and Wolf, A.: A mixed-integer program for drawing high-quality metro maps. Proc. 13th Internat. Sympos. Graph Drawing (GD'05).

[9] Stott, J. M. and Rodgers, P. : Metro Map Layout Using Multicriteria Optimization. In Proceedings of the Information Visualisation International 2004.

[10] Párraga, A. Benavente, R Vanrell, M. and Baldrich, R. Modelling inter-colour regions of Colour $\mathrm{Na}$ ming Space. In Proceedings on Color in Graphics, Imaging and Vision (CGIV'08). 